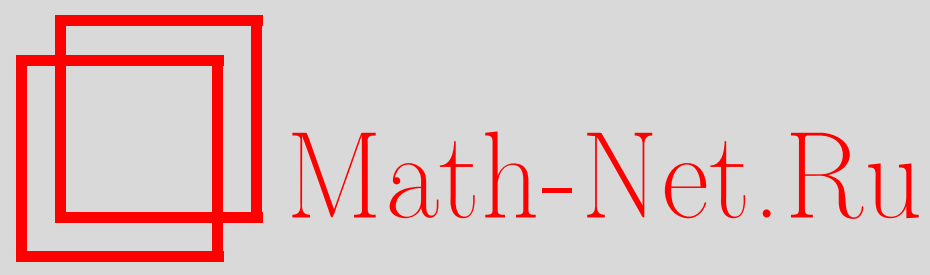

Ф. Н. Гарифьянов, Моменты Стильтьеса целых функций экспоненциального типа, Матем. заметки, 2000, том 67, выпуск 5, 674-679

DOI: https://doi.org/10.4213/mzm883

Использование Общероссийского математического портала Math-Net.Ru подразумевает, что вы прочитали и согласны с пользовательским соглашением http://www.mathnet.ru/rus/agreement

Параметры загрузки:

IP : 54.80 .97 .219

26 апреля 2023 г., $18: 26: 33$

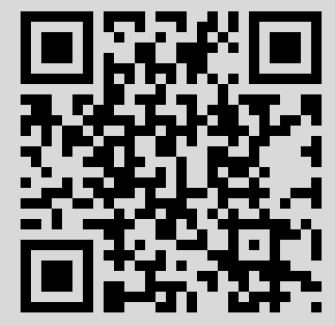




\section{МОМЕНТЫ СТИЛЬТЬЕСА \\ ЦЕЛЫХ ФУНКЦИЙ ЭКСПОНЕНЦИАЛЬНОГО ТИПА}

\section{Ф. Н. Гарифьянов}

Исследована лакунарная однородная проблема моментов

$$
\int_{0}^{\infty} F(x) e^{-x} x^{4 n+3} d x=0, \quad n=0,1, \ldots
$$

в классе целых функций экспоненциального типа.

Библиографоия: 10 названий.

1. Введение. Проблема моментов Стильтьеса (MC) состоит в отыскании функции $F(x)$, для которой

$$
\int_{0}^{\infty} F(x) x^{n} d x=c_{n}, \quad n=0,1, \ldots,
$$

где $c_{n}$ - заданные числа. Известно, что если $F(x) e^{\beta \sqrt{x}} \in L(0, \infty)$ при $\beta>0$, то $\exists n$ : $c_{n} \neq 0$. С другой стороны, для функций $F_{\mu}(x)=\exp \left(-x^{\mu} \cos \mu \pi\right) \sin \left(x^{\mu} \sin \mu \pi\right), 0<$ $\mu<1 / 2$, имеем $c_{n}=0 \forall n$ (см. работы Харди [1], [2] или их изложение в монографии Титчмарша [3, с. 406-409]). При $\mu=1 / 4$ отсюда следует, что у задачи

$$
\int_{0}^{\infty} F(x) e^{-x} x^{4 n+3} d x=0, \quad n=0,1, \ldots
$$

есть решение $F(x)=\sin x$. Это проверяется и непосредственно: достаточно рассмотреть интеграл от функции $z^{4 n+3} \exp (i z-z)$, взятьй вдоль вещественной оси от 0 до $R$, затем в положительном направлении вдоль окружности $|z|=R$ до мнимой оси и затем вдоль мнимой оси до начала координат, после чего перейти к пределу по $R \rightarrow \infty([4$, c. 115]). Очевидно, что справедлив и более общий результат: если $F(z)$-целая функция экспоненциального типа (конечной степени) $\sigma_{F}$ со свойством

$$
F(i z)=F(z)
$$

для индикатора которой выполнено неравенство

$$
h_{F}(0)<1
$$

то функция $\psi(x)=F(x) \sin x$-решение задачи (2). Неравенство (4) здесь играет двоякую роль. Во-первых, оно гарантирует существование $\mathrm{MC}$ целой функции $\psi(z) \exp (-z)$. 
Во-вторых, оно позволяет применить метод контурного интегрирования, поскольку тогда с учетом условия (3) и тригонометрической выпуклости индикатора $[4$, с. 193] выполняется неравенство $h_{F}(\theta)<\cos \theta+\sin \theta, \theta \in[0, \pi / 2]$. У целых функций экспоненциального типа со свойством (3) индикатор неотрицателен в силу принципа Фрагмена-Линделёфа, т. е. $\sigma_{\psi} \in[1,3 \sqrt{2} / 2)$. Кроме того, $\psi(\pi, k)=0, k=0, \pm 1, \pm 2, \ldots$

Возникает естественньй вопрос: существуют ли решения задачи $(2)$ с типом $\sigma<1$ ? Для ответа на него выясним, когда функция $F(x)$ сама удовлетворяет условиям (2). Другими словами, найдем такие дополнительные ограничения на функции $F(x)$, при выполнении которых задаче (2) одновременно удовлетворяют функции $F(x)$ и $F(x) \sin x$. Оказывается, что множество таких решений $F(x)$ типа $\sigma$ (в дальнейшем обозначенное через $\left.G_{\sigma}\right)$ непусто, если $\sigma \in[\sqrt{2} / 2, \sqrt{2})$. Метод исследования основан на сведении проблемы МС (2) к одному особому случаю теории линейных разностных уравнений с постоянньми коэффициентами, рассмотренному автором в статье [5].

2. Для построения решений из класса $G_{\sigma}$ удобно вместо условий $(2)$ взять равносильное им уравнение

$$
\int_{0}^{\infty} F(x) e^{-x}(\operatorname{sh} z x-\sin z x) d x=0, \quad|z|<1-h_{F}(0) .
$$

Применяя преобразование Бореля [6, с. 11-15]

$$
f(z)=\int_{0}^{\infty} F(x) e^{-z x} d x, \quad \operatorname{Re} z>h_{F}(0), \quad F(z)=\oint_{|\tau|=\sigma+\varepsilon} f(\tau) e^{z \tau} d \tau, \quad \varepsilon>0
$$

перейдем к уравнению для нижних функций

$$
f(1-z)-f(1+z)+i(f(1-i z)-f(1+i z))=0, \quad|z|<1-h_{F}(0) .
$$

Нижняя функция голоморфна при $|z|>\sigma$, исчезает на бесконечности и $f(i z)=-i f(z)$. $\mathrm{У}$ нее не менее четырех особых точек на окружности $|z|=\sigma$. Такой класс функций обозначим через $B_{\sigma}$. Уравнение (6) заменим разностным уравнением

$$
(V f)(z) \equiv \sum_{m=1}^{4} f\left(z+i^{m}\right)=0, \quad|z|<1-h_{F}(0)
$$

Наименьшее замкнутое вьпуклое множество $K$, содержащее все особенности функции $f(z)$ (сопряженная индикаторная диаграмма), инвариантно относительно поворота плоскости на прямой угол. В дальнейшем используем формулу

$$
h_{F}(\theta)=\max \operatorname{Re}\left(z e^{i \theta}\right), \quad z \in K \text {. }
$$

В зависимости от величины типа возможны следующие случаи.

а) $\sigma<\sqrt{2} / 2$. Множество $D=\mathbb{C} \backslash \bigcup_{m=1}^{4}\left(K+i^{m}\right)$ связно. Соотношение (7) вьполнено при $|z|>1+\sigma$, т.е. $f(z) \equiv 0[7$, с. 229$]$.

б) $\sigma=\sqrt{2} / 2$. Среди особенностей $f(z)$ есть и точки $\pm 1 / 2, \pm i / 2$-вершины квадрата $R$ (иначе множество $D$ связно). Соотношение (7) позволяет аналитически продолжить 
$f(z)$ в четыре сегмента, дополняюших $R$ до круга $|z|<\sqrt{2} / 2$. Следовательно, $\bar{R} \equiv K$. Однородное уравнение

$$
(V f)(z)=0, \quad z \in R,
$$

имеет нетривиальное решение $f(z) \in B_{\sqrt{2} / 2}\left[5\right.$, с. 15]. Его верхняя функция $F(z) \in G_{\sqrt{2} / 2}$, поскольку $(8) \Longrightarrow(7) \Longrightarrow(6) \Longrightarrow(5) \Longrightarrow(2)$. Стоит отметить, что $F(0) \neq 0$.

в) $\sqrt{2} / 2<\sigma<\sqrt{5} / 2$. Введем функцию $g(z)=\left(V f_{\sigma}\right)(z)$, где $f_{\sigma}(z)=4 z^{3}\left(z^{4}-z_{\sigma}^{4}\right)^{-1}$ и $\left|z_{\sigma}\right|=\sigma$. Она голоморфна в замькании $\bar{R}$, если

$$
\arcsin \frac{1}{2 \sigma}<\arg z_{\sigma}<\arccos \frac{1}{2 \sigma}
$$

Неоднородное разностное уравнение

$$
(V \widetilde{f})(z)=g(z), \quad z \in R
$$

разрешимо и $\widetilde{f}(z) \in B_{\sqrt{2} / 2}$, поскольку $g(i z)=-i g(z)[5$, с. 9]. Искомой нижней функцией будет разность $f(z)=\tilde{f}(z)-f_{\sigma}(z)$, так как квадрат с вершинами в точках $\pm z_{\sigma}$, $\pm i z_{\sigma}$ расположен слева от прямой $x=1 \Longrightarrow F(z) \in G_{\sigma}$.

г) $\sqrt{5} / 2<\sigma<\sqrt{2}$. Функция $F(z) \in G_{\sigma}$, если вместо условия (9) взять более сильное ограничение $\max \left[\operatorname{Re} z_{\sigma}, \operatorname{Im} z_{\sigma}\right]<1$.

д) $\sigma>\sqrt{2}$. Свойство (3) сразу влечет неравенство $h_{F}(0)>1$, и в силу известной теоремы В. Бернштейна [8, с. 99] MC целой функции $F(z) e^{-z}$ не существуют.

ЗАмЕчАниЕ 1 . Случай $\sigma=\sqrt{2}$, не исследованный в статье, представляет некоторьй интерес. Если в определении класса $G_{\sigma}$ неравенство (4) заменить на равенство $h_{F}(0)=1$, то все $\mathrm{MC}$ целой функции $F(z) e^{-z}$ могут существовать при вьполнении необходимого условия, что индикаторной диаграммой решения будет квадрат с вершинами в точках $\pm 1, \pm i$. Однако, используемьй выше метод построения нижней функции с полюсами на окружности $|z|=\sigma$ для $\sigma=\sqrt{2}$ уже неприменим.

Подведем итог.

Tеорема. Если $0<\sigma<\sqrt{2} / 2$ или $\sigma>\sqrt{2}$, mo $G_{\sigma}=\varnothing$. Если $\sigma=0$ или $\sqrt{2} / 2 \leqslant \sigma<\sqrt{2}$, то $G_{\sigma} \neq \varnothing$. При этом класс $G_{0}$ содержит лишь тривиальное решение $F(z) \equiv 0$, а класс $G_{\sqrt{2} / 2}$ совпадает с множеством функиий, ассоциированных по Борелю с множеством решений уравнения (8) из класса $B_{\sqrt{2} / 2}$.

3. Выясним, какими свойствами обладает построенная в случае б) целая функция $F(z) \in G_{\sqrt{2} / 2}$. Очевидно, что

$$
F(z)=\prod_{k=1}^{\infty}\left(1-\frac{z^{4}}{\alpha_{k}^{4}}\right)
$$

Получим некоторые результаты о распределении ее корней. Для этого воспользуемся интегральным представлением

$$
F(z)=\int_{\partial R} f^{-}(\tau) e^{z \tau} d \tau
$$


Приведем необходимые в дальнейшем сведения из статьи [5]. Нижняя функция $f(z)-$ это интеграл Коши

$$
f(z)=\frac{1}{2 \pi i} \int_{\partial R} \varphi_{0}(\tau)(\tau-z)^{-1} d \tau, \quad z \in \mathbb{C} \backslash \bar{R}
$$

аналитически продолжимый внутрь $R$ через любую из сторон квадрата и имеющий логарифмические особенности в его вершинах. Его плотность $\varphi_{0}(\tau)$ имеет точки разрьва первого рода в вершинах квадрата и бесконечно дифференцируема на замыкании каждой из сторон. У производных $\varphi_{0}^{(n)}(\tau)$ могут быть только точки разрьва первого рода в вершинах. Кроме того,

$$
\int_{\partial R} \varphi_{0}(\tau) d \tau \neq 0 ; \quad \varphi_{0}(i \tau)=-i \varphi_{0}(\tau) ; \quad \varphi_{0}[\alpha(\tau)]+\varphi_{0}(\tau)=0 .
$$

Здесь гомеоморфизм $\alpha(t): \partial R \rightarrow \partial R$ изменяет ориентацию, причем $\alpha(i \tau)=i \alpha(\tau)$ и на нижней стороне $\alpha(\tau)=\tau+i$. Таким образом, обратньй сдвиг $\alpha(\tau)$ разрьвен в вершинах, а в остальных точках $\alpha^{\prime}(\tau) \equiv 1$.

Формула Сохоцкого-Племеля

$$
f^{+}(\tau)-f^{-}(\tau)=\varphi_{0}(\tau)
$$

позволяет заменить в интеграле $(10)$ плотность $f^{-}(\tau)$ на $-\varphi_{0}(\tau)$. Но тогда

$$
F(z)=2 \cos \frac{z}{2} \int_{-1 / 2}^{1 / 2} \varphi_{1}(x) e^{z x} d x+2 \operatorname{ch} \frac{z}{2} \int_{-1 / 2}^{1 / 2} \varphi_{1}(x) \cos z x d x,
$$

где плотность $\varphi_{1}(x)=\varphi_{0}(x+i / 2)$ четна. Действительно, если точки $\tau_{1}$ и $\tau_{2}$ лежат на какой-то из сторон квадрата симметрично относительно его середины, то $\varphi_{0}\left(\tau_{1}\right)=\varphi_{0}\left(\tau_{2}\right)$. Это следует из нечетности плотности $\varphi_{0}(\tau)$ и последнего из условий $(11)$. В каждом из координатных углов у решения $F(z)$ тригонометрический индикатор $h_{F}(\theta)=\frac{1}{2}(\cos \theta+$ $\sin \theta), \theta \in[0, \pi / 2] ; h_{F}(\theta+\pi / 2)=h_{F}(\theta)$. Отсюда можно вывести следуюшие утверждения:

1) $F(z)=\operatorname{ch}(z / 2) F^{*}(z)+\cos (z / 2) F^{*}(i z)$, где четная функция $F^{*}(z)$ класса $A[9$, c. 100] и ее индикаторная диаграмма есть отрезок $(-i / 2, i / 2)$;

2) $F(z)$ - целая функция вполне регулярного роста с нулевой плотностью корней в каждом из координатных углов [8, с. 203], и почти для всех $\theta$ существует обычньй предел

$$
\lim _{r \rightarrow \infty} \frac{\ln \left|F\left(r e^{i \theta}\right)\right|}{r}=h_{F}(\theta),
$$

т. е. на таких лучах отсутствует исключительное множество нулевой относительной меры;

3) плотность корней $F(z)$ в любом угле $|\arg z-\pi m / 2|<\varepsilon$ не равна нулю, $m=0$, $1,2,3$.

Функции $F(z) \in G_{\sigma}, \sqrt{2} / 2<\sigma<\sqrt{2}$, построенные в статье, представимы в виде

$$
F(z)=\widetilde{F}(z)-\sum_{m=1}^{4} \exp \left(i^{m} z_{\sigma} z\right)
$$

Для первого слагаемого остаются в силе утверждения 1)-3), а индикатор $h_{F}(\theta)$ является индикатором квазиполинома. При $\sigma \rightarrow \sqrt{2}-0$ имеем $\arg z_{\sigma} \rightarrow \pi / 4$, причем всегда можно положить $\arg z_{\sigma}=\pi / 4$. 
4. Сделаем несколько замечаний об однородной проблеме $\mathrm{MC}$ в классе функций, голоморфных в плоскости с разрезом по лучу $(0,-\infty)$, обозначенной далее через $D_{1}$, и непрерьвно продолжимых на берега разреза, включая точку $z=0$. Под $\sqrt[k]{z}$ будем понимать ту ветвь радикала, которая голоморфна в $D_{1}$ и удовлетворяет условию $\sqrt[k]{x}>0$ при $x>0$. Если

$$
\int_{0}^{\infty} \Phi(x) x^{n} d x=0, \quad n=0,1, \ldots,
$$

то решениями задачи (13) будут и функции $\Phi(\sqrt[k]{\alpha x}), \alpha>0, k=1,2, \ldots$ Поэтому порядок решения $\rho$ в угле $(-\pi, \pi)$ определен с точностью до множителя $k^{-1}$, а тип и индикатор - с точностью до произвольного положительного множителя. Поясним, что порядок голоморфной в некотором угле функции понимается в смысле определения Титчмарша [4, с. 191], обобщенного Н. В. Говоровым [10, с. 10]. Тип, следуя [6, с. 9], определим формулой

$$
\sigma=\varlimsup_{r \rightarrow \infty}\left[r^{-\rho} \ln M_{-\pi, \pi}(r)\right], \quad M_{-\pi, \pi}(r)=\max _{-\pi \leqslant \theta \leqslant \pi}\left|\Phi\left(r e^{i \theta}\right)\right| .
$$

Возьмем частный случай функции Харди

$$
F_{1 / 4}(x)=\exp (-\sqrt[4]{\beta x}) \sin \sqrt[4]{\beta x}, \quad \beta>0
$$

и построенное в статье решение

$$
\Phi(x)=\exp (-\sqrt[4]{x}) P(x)
$$

где

$$
P(z)=\prod_{k=1}^{\infty}\left(1-\frac{z}{\alpha_{k}^{4}}\right)
$$

- целая функция вполне регулярного роста порядка $\rho=1 / 4$ и типа $\sigma=\sqrt{2} / 2$ с нулевой плотностью корней в каждом из углов $[-\pi, \varepsilon)$ и $(\varepsilon, \pi] \forall \varepsilon>0$. Постоянную $\beta$ в $(14)$ выберем так, чтобы $h_{F_{1 / 4}}(0)=h_{\Phi}(0)$. Весьма примечателен тот факт, что тогда

$$
h_{F_{1 / 4}}(\theta) \equiv h_{\Phi}(\theta)=\frac{1}{2}\left(\left|\sin \frac{\theta}{4}\right|-\cos \frac{\theta}{4}\right), \quad \theta \in[-\pi, \pi] .
$$

Итак оба решения (14) и (15) имеют порядок $\rho=1 / 4$, тип $\sigma=0$ и индикатор (16). Они голоморфны в $D_{1}$ и непрерывны в замыкании $\bar{D}_{1}$, но в их поведении на берегах разреза есть существенная разнища. Во-первых, $F_{1 / 4}(0)=0$, в то время как $\Phi(0)=1$. Во-вторых, для граничных значений функции (14) справедливы представления

$$
F_{1 / 4}^{\mp}(-x)= \pm(\exp (-\sqrt[4]{4 \beta x} i)-\exp (-\sqrt[4]{4 \beta x}))(2 i)^{-1}
$$

т. е. при $x \rightarrow \infty$ они осциллируют и $\left|F^{\mp}(-x)\right| \rightarrow 1 / 2$. Напротив, $\left|\Phi^{ \pm}(-x)\right|=O\left(|x|^{-1 / 4}\right)$; $\left|\Phi\left(r e^{i \theta}\right)\right|=O\left(\left|F_{1 / 4}\left(r e^{i \theta}\right)\right| r^{-1 / 4}\right)$, в чем легко убедиться, если применить в соотношении (12) формулу интегрирования по частям. Заметим, что при $\mu \in(1 / 4,1 / 2)$ индикатор функций $F_{\mu}(z)$ знакопеременен в угле $D_{1}$. 
ЗАмЕЧАнИЕ 2. Возьмем систему функций

$$
f_{4 m+3}(z):\left(V f_{4 m+3}\right)(z)=((4 m+3) !)^{-1} z^{4 m+3}, \quad z \in R .
$$

Функции, ассоциированные с ними по Борелю, обладают интересньм свойством

$$
\int_{0}^{\infty} F_{4 m+3}(x) e^{-x} x^{4 k+3} d x=\delta_{m, k} .
$$

Другими словами, существует система целых функций $P_{m}(z)$ порядка $\rho=1 / 4$ и типа $\sigma=\sqrt{2} / 2$, для которых вьполнены соотношения

$$
\int_{0}^{\infty} P_{m}(x) e^{-\sqrt[4]{x}} x^{k} d x=\delta_{m, k}
$$

ЗАМЕЧАНИЕ 3. Предложенный в статье метод позволяет исследовать и неоднородную проблему МC

$$
\int_{0}^{\infty} F(x) e^{-x} x^{4 k+3} d x=c_{4 k+3}
$$

при условии, что функция

$$
g(z)=\sum_{k=0}^{\infty} \frac{c_{4 k+3}}{(4 k+3) !} z^{4 k+3}
$$

голоморфна в $R$ и удовлетворяет условию Гёльдера на $\partial R$.

ЗАмЕчАниЕ 4. Сведением к разностным уравнениям можно исследовать и три другие проблемы $\mathrm{MC}$

$$
\int_{0}^{\infty} F(x) e^{-x} x^{4 m+k} d x=0, \quad m=0,1, \ldots, \quad k=0,1,2,
$$

в классе $G_{\sigma}$. Например, при $k=1$ для нижних функций имеем уравнение

$$
f(z+1)+f(z-1)-f(z-i)-f(z+i)=0, \quad z \in R,
$$

которое также было рассмотрено в статье [5].

\section{СПИСОК ЦИТИРОВАННОЙ ЛИТЕРАТУРЫ}

[1] Hardy G. H. On Stiltjes' "problème des moments" // Messenger of Math. 1917. V. 46. P. $175-182$.

[2] Hardy G. H. On Stiltjes' "problème des moments" // Messenger of Math. 1917. V. 47. P. $81-88$.

[3] Титчмарш Е. Введение в теорию интегралов Фурье. М.-Л.: ГИТТЛ, 1948.

[4] Титчмарш Е. Теория функций. М.: Наука, 1980.

[5] Гарифьянов $\Phi . Н$. Проблема обращения особого интеграла и разностные уравнения для функций, аналитических вне квадрата // Изв. Вузов. Матем. 1993. № 7. С. 7-16.

[6] Бибербах Л. Аналитическое продолжение. М.: Наука, 1967.

[7] Коробейник Ю. Ф. О решениях некоторых функциональных уравнений в классе функций, аналитических в выпуклых областях // Матем. сб. 1968. Т. 75. № 2. С. 225-234.

[8] Левин Б. Я. Распределение корней целых функций. М.: ГИТТЛ, 1956.

[9] Леонтьев А. Ф. Ряды экспонент. М.: Наука, 1976.

[10] Говоров Н. В. Краевая задача Римана с бесконечным индексом. М.: Наука, 1986. 